\title{
Comparing the Ultrasonography Diagnostic Value with Standard Radiography in Detecting Nasal Fractures
}

\author{
Research Article
}

\section{Mahyar Mohammadifard ${ }^{1}$, Ghasem Karimi2*, Afshin Eslamirad ${ }^{3}$, Ali Mohammad Poorbagher Shahri ${ }^{3}$, Mohammad Hasan Golbooyi}

\author{
1. Department of Radiology, Birjand University of Medical Science, Birjand, Iran \\ 2. Department of Otorhinolaryngology, Birjand University of Medical Science, Birjand, Iran \\ 3. Medical Intern, Birjand University of Medical Science, Birjand, Iran
}

\begin{abstract}
Background: Nasal bone fractures are the most typical kind of facial injuries. In recent years, ultrasonography has been suggested as an alternative method for detecting maxillofacial fractures. It can eliminate the risk of radiation exposure, especially in children and pregnant women. The objective of present study was to compare the diagnostic competency of conventional radiography with ultrasonography in the diagnosis of nasal injuries specially, fractures. Method: In prospective research, 331 patients with a history of midfacial trauma were enrolled. Following clinical examination, radiographs were obtained in lateral and occipitomental views. Ultrasonography was performed by an experienced sonologist, blinded to clinical findings in right and left lateral sides in addition to nasal dorsum. Images were analyzed by an experienced radiologist. Sensitivity, specificity and predictive values of radiology and US were determined by comparing their results with findings of the clinical examination as the gold standard. Results: In assessment of nasal fractures the sensitivity and specificity of ultrasonography were $97.79 \%$ and $97.66 \%$, respectively; while for radiography these two factors were $81.21 \%$ and $86.66 \%$, respectively. Based on the Chi-square test, ultrasonography was notably greater to radiography in diagnosing nasal fractures $(\mathrm{P}<0.001)$. Conclusion: Compared with radiography, ultrasonography had a higher sensitivity and specifity in detecting nasal fractures and seems to be an adequate method for the diagnosis of nasal fractures.
\end{abstract}

Keywords: Ultrasonography, Radiography, Diagnostic value, Nasal fractures.

\section{Introduction}

Nasal bone fractures are the most typical type of facial injuries. They constitute approximately $50 \%$ of all facial fractures. Inadequate treatment of nasal injury can cause complications like nasal deformity and dysfunction. Therefore, the accurate diagnosis of nasal fractures plays an important role in selecting appropriate treatment strategies. Clinical examination is considered as the gold standard in distinguishing the nasal fractures (1). Clinical criteria for nasal fractures are epistaxis, ecchymosis, deviation from midline, inflammation, instability, and crepitation (1, 2). However, association of nasal fractures with hematoma and edema makes the diagnosis difficult. Moreover, in some situations accurate imaging is essential because of possible legal consequences (3).

Even though conventional radiography is considered the standard imaging method for the diagnosis of nasal fracture and is commonly used by most clinicians, there are some discrepancies associated with it. As a result, many studies have investigated

*Corresponding Author:

\section{Ghasem Karimi,}

Department of Otorhinolaryngology,

Birjand University of Medical Science,

Birjand, Iran

Email: karimigh@bums.ac.ir substitute imaging techniques in the past years.

In recent years, ultrasonography (US) has been suggested as an alternative method for detecting maxillofacial fractures. It can eliminate the risk of radiation exposure, especially in children and pregnant women $(3,4)$. Although most studies have described US as a useful method for the initial detection of midface fractures, replacing conventional radiography by US is a feature that needs to be established (5).

The objective of present study was to compare the diagnostic competency of conventional radiography with US in the detection of nasal fractures.

\section{Materials and Methods}

In this prospective research,331 patients with a history of midfacial trauma who were referred Emergency Department of Imam Reza Hospital, Birjand, Iran from August 2017 to August 2018 were enrolled. Informed consent was obtained from all patients after explanation of study process. Pregnant women and children under age 18 were excluded from the study.

Following clinical examination, radiographs were obtained in lateral and occipitomental views of nasal bones.

Immediately or within 24 hours after radiography, US was performed in the supine position by an experienced radiologist, blinded to clinical findings, using 10-MHz ultrasound head (Esaote, North America, 
ultrasound MyLab ${ }^{\text {TM} 50 ~ X ~ V i s i o n) ~ i n ~ r i g h t ~ a n d ~ l e f t ~}$ lateral sides in addition to the nasal dorsum.

Radiographs were analyzed in a masked manner by the same radiologist who performed the real time ultrasound. Radiographic and ultrasound findings were compared with clinical examination results as the gold standard for sensitivity, specificity, and predictive values.

Data analysis was performed with SPSS software, version 15. Assessing the significant differences was done by Chi-square test, considering the $\mathrm{P}<0.05$ as significant value.

\section{Results}

331 patients (103 women and 228 men) with the age of $31.39 \pm 10.06($ mean \pm SD) were included in this study. Based on physical examination nasal bone fractures were diagnosed in $181(54.7 \%)$ of the 331 patients. The most common reasons for nasal fractures are traffic collision and fights. Closed reduction was performed in all fractured nasal bones within 14 days after trauma.

Radiography diagnosed fractures in 147 of 181 clinically confirmed cases of nasal fractures with sensitivity and specificity of $81.21 \%$ and $86.66 \%$, respectively. US revealed nasal fractures in 177 of 181 patients. The sensitivity and specificity of US in diagnosis of nasal fractures was $97.79 \%$ and $96.66 \%$ respectively. The Chi-square test showed that US was significantly greater to radiography in diagnosis of nose bone fractures $(\mathrm{P}<0.001)$. The findings of the analysis are detailed in table 1.

Table 1: Comparing the negative and positive predictive values plus sensitivity and specificity of radiography and sonography

\begin{tabular}{|l|l|l|l|l|}
\hline Diagnostic method & Sensitivity\%(n) & Specificity \%(n) & $\begin{array}{l}\text { Negative predictive } \\
\text { value\%(n) }\end{array}$ & $\begin{array}{l}\text { Positive predictive } \\
\text { value\%(n) }\end{array}$ \\
\hline Radiography & $81.21(147 / 181)$ & $86.66(130 / 150)$ & $79.26(130 / 164)$ & $88.02(147 / 167)$ \\
\hline Ultrasonography & $97.79(177 / 181)$ & $96.66(145 / 150)$ & $97.31(145 / 149)$ & $97.25(177 / 182)$ \\
\hline
\end{tabular}

\section{Discussion}

The present study revealed that US can diagnose nasal fractures with high sensitivity and specificity. Based on our results US was significantly better than radiography in the detection of nasal fractures.

Conventional radiography as a standard imaging method is usually the initial step for assessment of suspected nasal fractures. Because suspected cases of nasal bone fractures with midface trauma usually need complete evaluation of other parts of the facial skeleton, taking radiographs for detecting associated bone fractures of the craniofacial skeleton is critical especially if computed tomography (CT) scan is not available (6). But practically radiography has low diagnostic value in detecting nasal fractures and diagnoses are often made based on clinical examination $(7,8)$. Similar to previous studies our results showed the high sensitivity of radiography in detecting nasal fractures. However, it has been found that conventional radiography has only high sensitivity in diagnosis of nasal dorsum fractures and its sensitivity in detecting lateral nasal wall fractures is low $(9,10)$.

In recent years some studies have suggested the US as a safe and accurate imaging method for diagnosis of maxillofacial fractures. In the study by Hong et al US could detect all fracture lines in children with nasal trauma. Their results showed that US can evaluate the cartilaginous septum while the radiography and computed tomography (CT) scan have limited diagnostic ability (11). Thiede et al found that US has greater sensitivity and specifity in detecting lateral nasal wall fractures compared with radiography. They suggested ultrasound as an alternative imaging method to radiography (10). Lee et al reported that highresolution US is able to show all nasal fractures (12).
Atighechi et al compared the diagnostic value of ultrasound with radiography in detection of nasal fractures. Their results showed that ultrasound can be a good substitute for radiography in early diagnosis of nose injuries mainly fractures (16). Current study confirmed the results of Atighechi's research group.

US is a fast, available and non-invasive method which eliminates the risk of $\mathrm{x}$-ray exposure. Because of real-time image visualization it makes the adequate evaluation of fracture easier. But some limitations have been considered for US including dependency on operator experience $(5,13)$, lack of ability to detect complex maxillofacial fractures, and limited potential in diagnosis of non-displaced fractures of most facial bones (13-15). In this situation CT can be useful in addition to US.

The main limitation of the current study is that we did not compare the two diagnosis methods based on fracture locations. It seems that in this way we could have a more accurate interpretation from the results of the study.

Conclusively, our results suggest that the US has a higher sensitivity and specificity compared with conventional radiography and can lead the clinician rapidly and efficiently to an accurate diagnosis.

\section{References}

1. Chan J, Most SP. Diagnosis and management of nasal fractures. Operative Techniques in Otolaryngology-Head and Neck Surgery. 2008;19 (4):263-6.

2. Kelley BP, Downey CR, Stal S. Evaluation and reduction of nasal trauma. Semin Plast Surg. 2010;24(4):339-347. 
3. Yoon HY, Han DG. Delayed Reduction of Nasal Bone Fractures. Arch Craniofac Surg. 2016;17 (2):51-55.

4. Javadrashid R, Khatoonabad M, Shams N, Esmaeili F, Jabbari Khamnei H. Comparison of ultrasonography with computed tomography in the diagnosis of nasal bone fractures. Dento maxillo facial radiology. 2011;40(8):486-91.

5. Adeyemo WL, Akadiri OA. A systematic review of the diagnostic role of ultrasonography in maxillofacial fractures. International journal of oral and maxillofacial surgery. 2011;40(7):655-61.

6. Schuknecht B, Graetz K. Radiologic assessment of maxillofacial, mandibular, and skull base trauma. European radiology. 2005;15(3):560-8.

7. Logan M, O'Driscoll K, Masterson J. The utility of nasal bone radiographs in nasal trauma. Clinical radiology. 1994;49(3):192-4.

8. Pérez-Guisado J, Maclennan P. Clinical evaluation of the nose: a cheap and effective tool for the nasal fracture diagnosis. Eplasty. 2012;12:e3.

9. Gurkov R, Clevert D, Krause E. Sonography versus plain $\mathrm{x}$ rays in diagnosis of nasal fractures. American journal of rhinology. 2008;22(6):613-6.

10. Thiede O, Krömer J, Rudack C, Stoll W, Osada N, Schmäl F. Comparison of ultrasonography and conventional radiography in the diagnosis of nasal fractures. Arch Otolaryngol Head Neck Surg. 2005;131(5):434-9.
11. Hong HS, Cha JG, Paik SH, Park SJ, Park JS, Kim $\mathrm{DH}$, et al. High-resolution sonography for nasal fracture in children. AJR American journal of roentgenology. 2007;188(1):86-92.

12. Lee M, Cha J, Hong H, Lee J, Park S, Paik S, et al. Comparison of high-resolution ultrasonography and computed tomography in the diagnosis of nasal fractures. J Ultrasound Med. 2009;28(6):717-23.

13. Morris EC, Rebello P, Thomson KJ, Peggs KS, Kyriakou C, Goldstone AH, et al. Pharmacokinetics of alemtuzumab used for in vivo and in vitro T-cell depletion in allogeneic transplantations: relevance for early adoptive immunotherapy and infectious complications. Blood. 2003;102(1):404-6.

14. Jank S, Deibl M, Strobl H, Oberrauch A, Nicasi A, Missmann $M$, et al. Interrater reliability of sonographic examinations of orbital fractures. European journal of radiology. 2005;54(3):344-51.

15. Jank S, Emshoff R, Etzelsdorfer M, Strobl H, Nicasi A, Norer B. Ultrasound versus computed tomography in the imaging of orbital floor fractures. Journal of oral and maxillofacial surgery : official journal of the American Association of Oral and Maxillofacial Surgeons. 2004;62(2):150-4.

16. Atighechi S, Baradaranfar $\mathrm{MH}$, Karimi G, Dadgarnia MH, Mansoorian HR, Barkhordari N, et al. Diagnostic value of ultrasonography in the diagnosis of nasal fractures. The Journal of craniofacial surgery. 2014;25(1):e51-3. 